\title{
Multilevel Computational Model for Cost and Carbon Optimisation of Reinforced Concrete Floor Systems
}

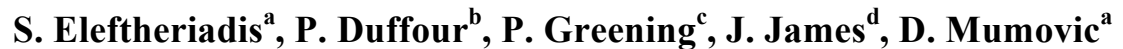 \\ ${ }^{a}$ UCL Institute for Environmental Design and Engineering, University College London, UK \\ ${ }^{b}$ Department of Civil, Environmental \& Geomatic Engineering, University College London, UK \\ ${ }^{c}$ Faculty of Engineering, Environment and Computing, Coventry University, UK \\ ${ }^{d}$ JAW Sustainability, UK \\ E-mail: stathiseleftheriadis@gmail.com
}

\begin{abstract}
The cost and carbon efficiency of building structures could be enhanced by the current developments in design automation and optimisation techniques. New ways to systematically assess design alternatives based on cost and carbon parameters are necessary. The study proposes a multilevel optimisation approach that combines Building Information Modelling (BIM) data and Finite Element Modelling (FEM) with a constrained genetic algorithm. The optimisation methodology is tested in a prototypical building floor system. Structural grid configurations, floor thicknesses and columns sizes and reinforcement details are identified. The results showed that the cost optimum design is $3 \%$ cheaper than the carbon optimum design but it has $7 \%$ more carbon. In addition, the concrete in the floor is the biggest contributor in both total cost and carbon. Relationships between cost- and carbon- optimum designs for the tested structural configuration are also discussed.
\end{abstract}

Keywords -

Optimisation; Reinforced Concrete; Cost; Carbon; BIM; FEM

\section{Introduction}

In reinforced concrete (RC) structures, cost optimisation has traditionally attracted attention amongst researchers and practitioners due to the increasing demands on the cost efficiency of buildings' design and construction [1]. Cost optimisation studies have analysed common structural systems including beams, columns, frames and floors. An exhaustive survey of cost optimisation methods in various RC structures can be found in Sarma \& Adeli [2].

In flat slab systems, which are one of the most popular solutions in commercial RC buildings in the UK, only a few previous studies were found. Sahab et al. [3] applied genetic algorithms using the Equivalent Frame Method (EFM) to optimise the floors in one-storey, three-storey and four-storey buildings. In their study, column layout optimisation could significantly reduce the total cost of the structure. However, the algorithm was based on EFM method, which could limit the applications in more complex building scenarios.

Only recently, Aldwaik \& Adeli [4] have proposed a Finite Element Model (FEM) cost optimisation method for flat slabs using neural dynamics. They have reported cost savings of approximately $7-9 \%$ when compared the original design configurations for a floor scenario in a real-life high-rise building. Despite the interesting results, their study did not include any layout optimisation which could potentially offer larger savings. Environmentally-based optimisation studies in $\mathrm{RC}$ structures are far less developed than the cost-based ones. Nevertheless, it is becoming more challenging to make cost-effective decisions without evaluating the relationships between the economic and the environmental parameters together [5].

Generally, the implementation of environmentallybased optimisation in structural engineering could be restricted by the: 1) Type of the objective functions not being universally agreed, 2) Constructability constraints not adequately addressed or included in the optimisation model [5]. A few optimisation studies that inlclude both cost and carbon parameters involve concrete beams [6][11], frames [7][8] and columns [9][10]. To our best knowledge there is no flat slab study with these two objectives.

The use of Building Information Modelling (BIM) has the potential to improve the way environmental and cost models are integrated within building system analysis $[14,15]$. The current study extends Aldwaik \& Adeli's [4] FEM-based model and explores how BIM technologies could also be combined with Finite Element Models (FEM) to develop cost and carbon optimisation models that can also be implemented in real-life building structures. The aim of the study is to 
introduce a computational model that systematically tackles cost and carbon optimisation problems in $\mathrm{RC}$ flat slab buildings. The objectives of the study are threefold:

1. To establish a multilevel approach for the optimisation of flat slabs based on cost and carbon functions,

2. To utilise data inputs from Building Information Model (BIM) and a Finite Element Model (FEM) engine, and

3. To validate the proposed optimisation methodology in a prototypical case study scenario and analyse the findings.

\section{Methodology}

Figure 1 shows the flow diagram of the methodology used in this study. The optimisation process begins by obtaining floor information from BIM components including node coordinates and total floor area. BIM data are then used as the input to create the geometric constraints of the structural floor in the FEM component. The structural analysis of the proposed optimisation methodology is performed in three main levels:

1. Structural grid layout - number and size of bays

2. Structural design elements - floor and columns dimensions

3. Structural detailing - floor and columns reinforcement

The reason for this distinction is to identify the parameters that have the biggest contribution in the structure's embodied carbon and construction cost. The optimisation engine is a custom constrained genetic algorithm with penalisation factoring. The formulation of the objective functions is based on construction cost and embodied carbon inventories which were collected from literature sources. Two separate single-objective modes of the algorithm were built with: 1) A cost objective function, and 2) A carbon objective function.

The multiobjective functionality of the model is also under development but it is not covered in this study. The optimisation component utilises FEM to run and perform the structural verifications based on Eurocode 2 requirements. The genetic algorithm searches in the design space for the solutions that minimise the objective functions whilst satisfying the structural code's requirements and constructability constraints. Once the genetic algorithm operator is minimised the optimisation process ends and the optimised flat slab configurations are obtained. In the subsequent sections the main components of the methodology are presented.

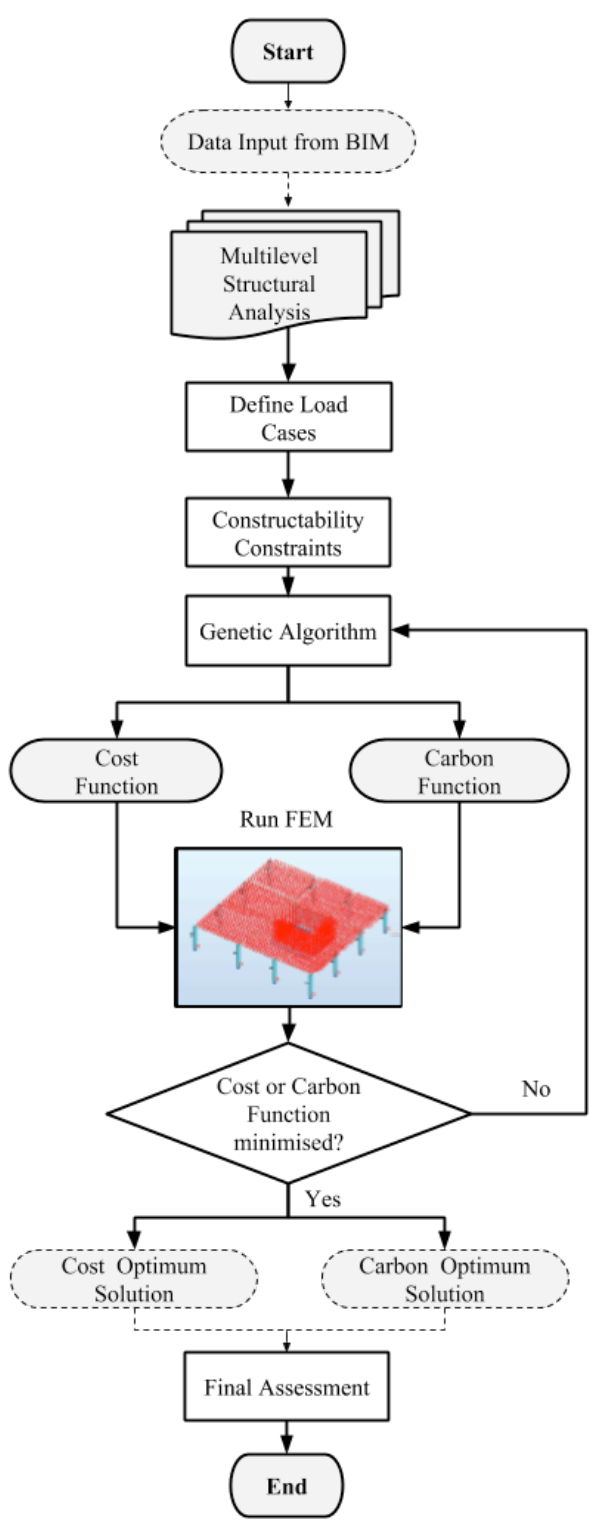

Figure 1. Flow diagram showing the proposed methodology with its main functions

\subsection{BIM Component}

Obtaining high level of BIM integration in structural analysis is still considered a significant challenge in the current design practice. In this study, BIM functionalities are incorporated in the optimisation model. In particular, data required for the optimisation in FEM are directly obtained from the BIM geometry. The topology of the flat slab construction is mapped in BIM and transferred into the optimisation module where all coordinates, dimensions and material properties are recognised and translated into structural panels. To enhance the interoperability of the BIM model with the FEM engine in this study, Autodesk Revit 2016 is used 
as the BIM model and Autodesk Robot 2016 as the FEM analysis engine. More information on BIM applications can be found in previous literature review where it was suggested that more than $70 \%$ of recent studies use Autodesk Revit for BIM-based life cycle and sustainability analysis of building systems [15].

This type of FEM offers parametric modelling features, which are necessary when conducting multiple iterations for the verification of the genetic algorithm. Robot Application Programming Interface (API) gives access to the functionalities of the Robot programme and to individual components of the modelled structure from the programming language level. In this research, C\# was used to access the .NET framework of Robot API and to design the optimisation based on its Windows Form Application. The programmable COM interface and the API of Robot automate repetitive tasks in Robot such as multiple case scenarios.

\subsection{Genetic Algorithm}

A tailored genetic algorithm was developed in $\mathrm{CH}$ to optimise the structure. The genetic algorithm's conceptual construct, developed by Holland [12] in the 1960 's and 1970's mimics the evolutionary processes in nature by population, reproduction and heredity with the inherent ability for the designer to alter several parameters within the method such as population size, crossover technique and mutation rate. Figure 2 gives a schematic representation of the single-objective GA process implemented in this study.

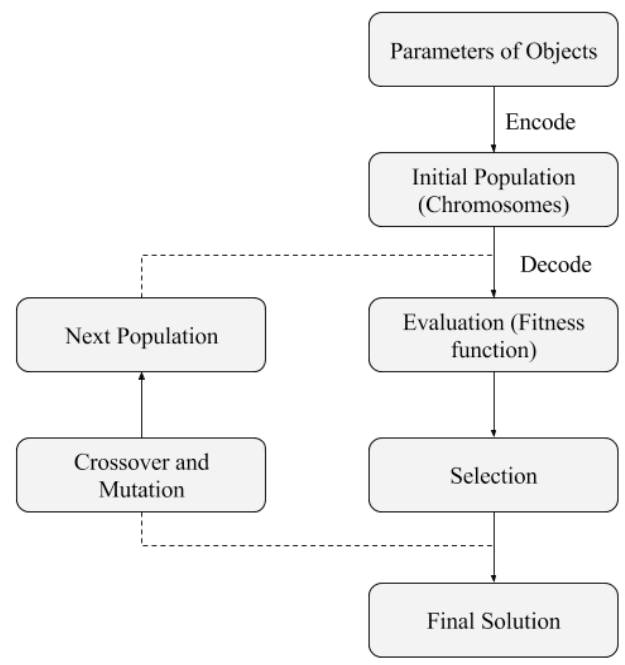

Figure 2. Schematic of single objective genetic algorithm (adapted from: [13])

Two different objective functions are utilised in the genetic algorithm to minimise the construction costs and embodied carbon of the whole structure.

\subsubsection{Cost Function}

The fitness function used in the calculation of the structural cost is modified to include the construction costs of the floor and columns. Equation (1) shows the general representation of the cost function used in the study.

$$
f_{c}(x)=\sum_{i=0}^{n} q_{i} c_{i}
$$

where $q_{i}$ represents quantities (material weights) obtained from the FEM structural analysis and $c_{i}$ represents the cost factors for the different material types (concrete, steel reinforcement and formworks). The sum of the different structural members costs (columns and floor) is the total cost of the structure. Material and labour cost data are collected from Spon's Architects' and Builders' Price Book 2017. As the cost factors have different units appropriate conversion factors were used. The output from the cost function is a total $£$ cost. The final result is weighted in $£ / m^{2}$ of floor area.

\subsubsection{Carbon Function}

The fitness function that calculates the embodied carbon of the structural elements in the genetic algorithm is shown in Equation (2).

$$
f_{e c}(x)=\sum_{i=0}^{n} q_{i} e_{i}
$$

where $q_{i}$ is the quantities (material weights) obtained from the FEM structural analysis and $e_{i}$ is the carbon factors for concrete, reinforcement. It follows a similar procedure with the cost function but the cost factors are substituted with the relevant carbon factors for the different material types. The carbon factors are based on Environmental Product Declaration (EPD) data. For the concrete carbon factor $130 \mathrm{kgCO}_{2} \mathrm{e} / \mathrm{t}$ was assumed and $1270 \mathrm{kgCO}_{2} \mathrm{e} / \mathrm{t}$ was considered for the steel carbon factor. The final carbon results were converted in $\mathrm{kgCO}_{2} \mathrm{e} / \mathrm{m}^{2}$ based on the total floor area.

\subsection{Optimisation Levels}

The optimisation of the structure is organised around three main levels: 1) Structural layout, 2) Sizes of columns and slabs, and 3) Reinforcement layout for columns and slabs. The components on these three levels are interconnected and therefore, understanding how these relationships affect the the cost and carbon performance of the structure becomes critical.

Several computational modules have been established to ensure the seamless integration of these levels with the optimisation algorithm. The sections below elaborate on the integration process. 


\subsubsection{Grid Layout}

In this level the algorithm looks for the structural grid topology that minimises the objective functions. A computational module was created to enable the automatic generation of structural grids - i.e. columns location. The floor outline is obtained from the BIM model, and it is used to determine the boundaries of the slab. The algorithm reads the relevant boundary lengths and uses them to calculate all the possible structural bay combinations on $\mathrm{X}$ - and $\mathrm{Y}$ - directions. The algorithm uses a polynomial time approximate component and permutation with repetition to find all the appropriate bay combinations. The user assigns possible span lengths, which can be any integer, double or float number. In this research, a defined set of span lengths was used to resemble realistic conditions: The algorithm uses $0.5 \mathrm{~m}$ length increments.

\subsubsection{Column and Slab Sizing}

The optimum sizing of the slab is determined by varying the slab depth within a discrete set of dimensions. This set includes constructability constraints. For example, for the slab thickness, the allowable limits have been constrained to practical increments of $25 \mathrm{~mm}$. The data are encoded into input arrays, and the algorithm uses a random operator to select an element from the list during each iteration. Discrete variables are used for the dimension optimisation of the RC columns. For this operation, $50 \mathrm{~mm}$ increments are implemented.

\subsubsection{Detailing}

Detailing parameters include all the reinforcement elements in the slab and the columns. The slab reinforcement (both $\mathrm{X} \& \mathrm{Y}$ directions) consists of a basic mesh that is applied everywhere with additional top and bottom reinforcement bars only applied on the zones that are needed. For the columns, bending reinforcement is considered. All the designs are compliant with the Eurocode's requirements. This set of analysis consists of several algorithmic components.

For the slab, the algorithm reads the required reinforcement data (Figure 3) from all the mesh points in the FEM model and it calculates the minimum and the maximum values. The minimum values are used for the estimation of the basic mesh and the maximum values are used for the calculation of the additional reinforcement where necessary. The bar diameters are limited to the most common diameters engineers use in practice $(\varnothing 10, \varnothing 12, \varnothing 16, \varnothing 20, \varnothing 25, \varnothing 32)$. Their spacing also follows the common increments of $175 \mathrm{~mm}, 200 \mathrm{~mm}$, $225 \mathrm{~mm}$ and $250 \mathrm{~mm}$.

The total quantities (in tonnes) of basic reinforcement is then calculated by multiplying the reinforcement rate with the area of the slab. The differences between the assigned basic reinforcement and the maximum mesh/node values of the required reinforcement are used to estimate the area of the additional bars. Each component of the mesh carries a digital identity which is used in this component in order to identify the zones in need of additional reinforcement.

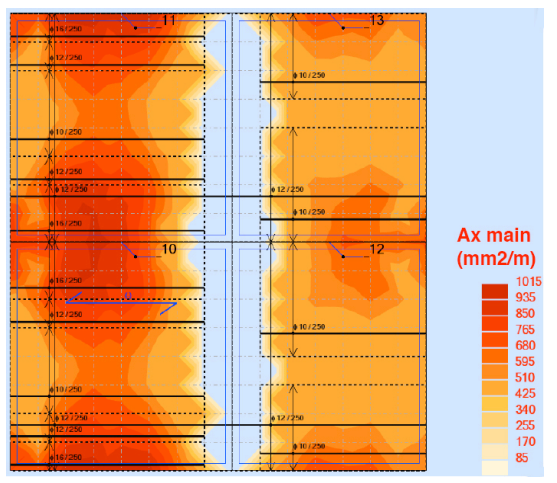

Figure 3. Required reinforcement mapping on the flat slab

A separate algorithmic component is implemented for the calculation of the bending reinforcement in the columns. This component uses forces (reaction loads), geometric (column cross section, storey height) and material data from the structural FEM model. Structural checks are conducted and the approved reinforcement is obtained from the calculation spreadsheet. The material quantities for the columns are used to update the objective function of the optimisation.

\section{$3 \quad$ Prototype Building}

\subsection{Geometric Conditions}

The optimisation methodology described in the previous sections is tested on a prototype building scenario. Figure 4 shows the simplified plan of the floor for the tested building scenario as displayed in BIM. The floor consists of a $15 \times 16 \mathrm{~m}$ rectangle area with a centralised core structure for lateral stability. For the purposes of this study, the core structure $(4 \times 5 \mathrm{~m})$ is a fixed component assuming a RC $200 \mathrm{~mm}$-thick wall.

The functional units for the objective functions are the slab and the columns. The dimensions of the floor are also fixed which means that the columns' topology will be fitted within the boundary of the floor. It is envisaged that this type of geometry can be implemented in real-life high-rise buildings. In regards to the materials properties, $\mathrm{C} 32 / 40$ is used for the concrete $\left(2400 \mathrm{~kg} / \mathrm{m}^{3}\right)$ and S500 for the steel bars $(7850$ $\mathrm{kg} / \mathrm{m}^{3}$ ). For the loads, it is assumed $2.5 \mathrm{kN} / \mathrm{m}^{2}$ was assumed for superimposed load, $7.5 \mathrm{kN} / \mathrm{m}^{2}$ for live load and the dead load of the structure. 


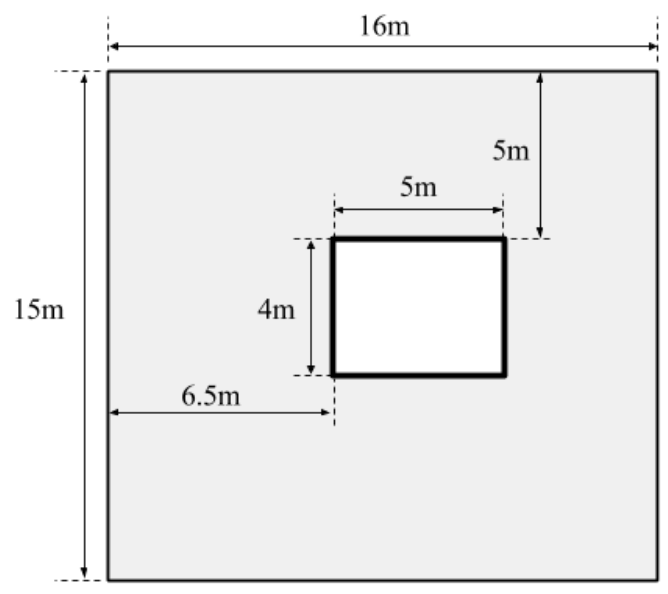

Figure 4. Plan of the floor boundary conditions

\subsection{Algorithmic Input}

Table 1 shows the input variables that are used in this building scenario. The algorithm uses the values from that list to find the combinations of genes that minimise either the cost or the carbon functions. A population of 100 individuals is used in the algorithm whilst the maximum generations were limited to 350 generations.

Table 1. Gene components for the genetic algorithm

\begin{tabular}{|c|c|c|c|}
\hline Gene & Type & $\begin{array}{l}\text { Number } \\
\text { of Options }\end{array}$ & Designs \\
\hline 1 & $\begin{array}{c}\text { Slab } \\
\text { Thickness }\end{array}$ & 5 & $\begin{array}{c}\{225,250,275,300 \\
325\} \mathrm{mm}\end{array}$ \\
\hline 2 & Column B & 4 & $\begin{array}{c}\{250,300,350,400\} \\
\mathrm{mm}\end{array}$ \\
\hline 3 & Column H & 4 & $\begin{array}{c}\{250,300,350,400\} \\
\mathrm{mm}\end{array}$ \\
\hline 4 & Bays X & $4 *$ & $\begin{array}{l}\{(5 \times 6 \times 5),(6 \times 5 \times 5) \\
(8 \times 8),(5 \times 5 \times 6)\} \mathrm{m}\end{array}$ \\
\hline 5 & Bays Y & $4^{*}$ & $\begin{array}{c}\{(5 \times 5 \times 5),(8 \times 7), \\
(7.5 \times 7.5),(7 \times 8) \mathrm{m}\end{array}$ \\
\hline 6 & $\begin{array}{l}\text { Bars per } \\
\text { Column B }\end{array}$ & 3 & $\begin{array}{c}\{3,4,5\} \text { Number of } \\
\text { Bars }\end{array}$ \\
\hline 7 & $\begin{array}{l}\text { Bars per } \\
\text { Column } \mathrm{H}\end{array}$ & 3 & $\begin{array}{c}\{3,4,5\} \text { Number of } \\
\text { Bars }\end{array}$ \\
\hline \multicolumn{4}{|c|}{ Combinations } \\
\hline \multicolumn{4}{|c|}{$\begin{array}{l}\text { *As generated by the computational compoment: The } \\
\text { Number of options depends on the inputs' details. In } \\
\text { this case, values for the bays between } 5 \mathrm{~m} \text { to } 9 \mathrm{~m} \text { with } \\
0.5 \mathrm{~m} \text { increments are used. }\end{array}$} \\
\hline
\end{tabular}

\subsection{Penalisation}

The structural calculations are based on EN 1992-11:2004 AC:2008. If the limit states defined in the code are violated, the algorithm assigns a penalty in the objective functions previously described. The design inputs that cause the violation receive lower probability for appearing in the future generations and they are eventually are eliminated from the population. Other structural constraints such as deflection limits could also be incorporated in the penalty function. This penalisation approach is an efficient way to assist the algorithm in finding the optimum combinations faster.

\section{Results}

\subsection{Algorithmic Performance}

Several optimisation sequencies (at least 10 runs for every objective) were run and in every instance the algorithm converged to the maximum performance after approximately 280 generations. Figure 5 shows the representation of the algorithms' convergence pattern displaying continuous improvements on the fitness function after each generation. Each iteration took approximately 25-30 seconds to complete in an Intel Corei5-4570@3.2 GHz desktop computer.

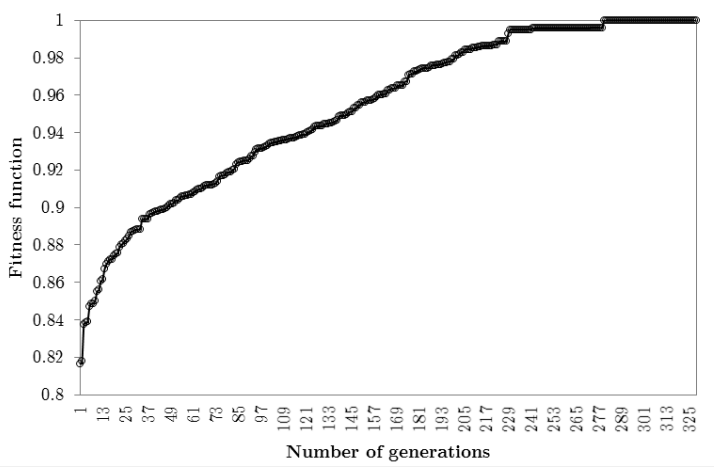

Figure 5. Convergence curve of the optimisation algorithm

An important component of the algorithm is its ability to accurately calculate the reinforcement rates in the slab. The zoning module effectively identifies the variability of slab thickness and the reinforcement requirements. Figure 6 demonstrates the relationship between the tonnage of steel and concrete for the different slab thicknesses in a simple way. Two observations can be made: 1) Thinner slabs need more reinforcement than thicker ones, 2) The options with the $300 \mathrm{~mm}$ and $325 \mathrm{~mm}$ slabs do not provide significant savings in reinforcement due to high minimum reinforcement requirements. For the analysis of the results, the entire optimisation set the input and output data categories were exported in a .csv file. The analysis of the results was conducted using Excel. 


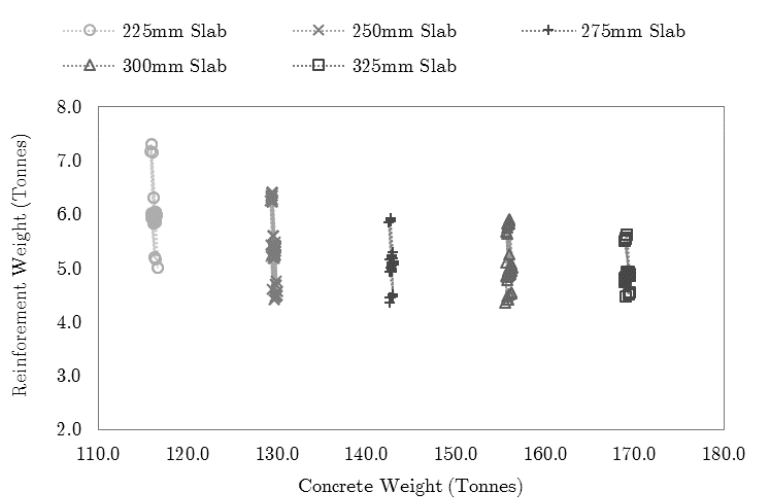

Figure 6. Relationship between concrete weight and reinforcement weigth in the slab

\subsubsection{Carbon Performance}

The performance of the algorithm for the carbon function is summarised in Figure 7. It becomes evident that the concrete in the slab is the biggest contributor to the total embodied carbon in the structure as the two curves appear to have very similar patterns. The reinforcement in the slab is the second biggest contributor. On average $90 \%$ of the carbon in the structure is attributed to the slab materials.

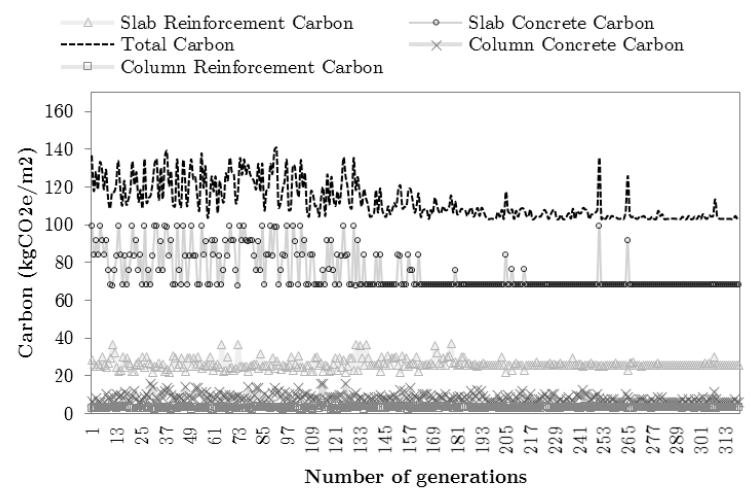

Figure 7. Evolution of carbon components towards the carbon optimum solution

\subsubsection{Cost Performance}

Figure 8 shows the performance of the algorithm for the cost function. The initial fluctuations correspond to the early stages of the search, however the algorithm eventually stabilises around $120 £ / \mathrm{m}^{2}$. In this set of simulations, no single parameter clearly affects the results unlike in the carbon optimisation. Nevertheless, the slab concrete and formwork are the most contributing to the structure's total cost. Detailed cost breakdown for the cost optimum solutions will be presented in the following sections.

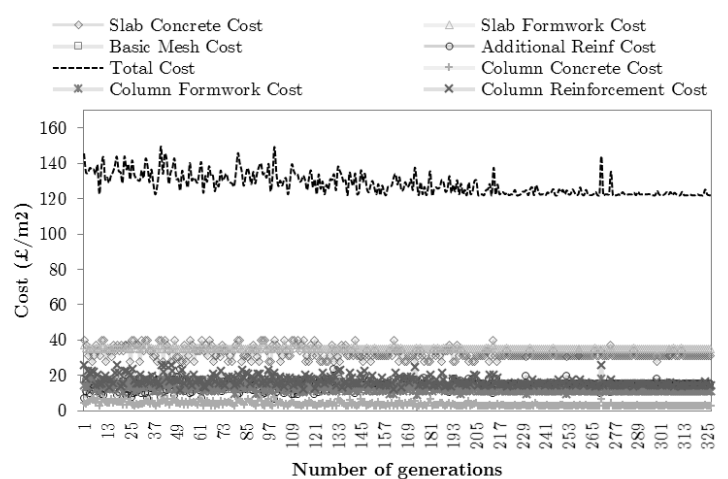

Figure 8. Evolution of cost components towards cost optimum solution

\subsection{Optimisation Solutions}

In this section, the optimum solutions obtained from both of the objective functions are presented. Table 2 summarises the results. The algorithm search has been simplified for the design of the columns by applying the same dimensions on every column (the reinforcement calculation applies the same bar diameter to every bar in the section). If required, both elements could be overriden to allow for further customisation. Figure 9 shows the geometric representation of the optimum flat slab designs. The carbon optimum solution has more columns (12 in total) and thinner slabs $(225 \mathrm{~mm}$ slab thickness). This finding verifies the relationship between the carbon function and the slab concrete as the algorithm tries to minimise it. The additional columns reduce the reinforcement requirements in the slab and as a result, the total carbon of the structure.

Table 2. Optimisation results

\begin{tabular}{ccc}
\hline & $\begin{array}{c}\text { Carbon } \\
\text { Optimum }\end{array}$ & $\begin{array}{c}\text { Cost } \\
\text { Optimum }\end{array}$ \\
\hline Slab Thickness & $225 \mathrm{~mm}$ & $250 \mathrm{~mm}$ \\
Column B & $250 \mathrm{~mm}$ & $250 \mathrm{~mm}$ \\
Column H & $250 \mathrm{~mm}$ & $250 \mathrm{~mm}$ \\
Bays X & $6 \mathrm{mx} 5 \mathrm{mx} 5 \mathrm{~m}$ & $5 \mathrm{mx} 6 \mathrm{mx} 5 \mathrm{~m}$ \\
Bays Y & $5 \mathrm{mx} 5 \mathrm{mx} 5 \mathrm{~m}$ & $8 \mathrm{mx} 7 \mathrm{~m}$ \\
Columns & $8 \mathrm{Bars} \mathrm{per}$ & $8 \mathrm{Bars} \mathrm{per}$ \\
Reinforcement & Column & Column \\
Total number of & $12(5 \times \varnothing 16$, & $10(1 \mathrm{x} \varnothing 20$, \\
Columns & $7 \times \varnothing 12)$ & $3 \times \varnothing 16,6 \times \varnothing 12)$ \\
Slab & $101 \mathrm{~kg} / \mathrm{m}^{3}$ & $98 \mathrm{~kg} / \mathrm{m}^{3}$ \\
Reinforcement & & \\
Rates & & \\
\hline
\end{tabular}



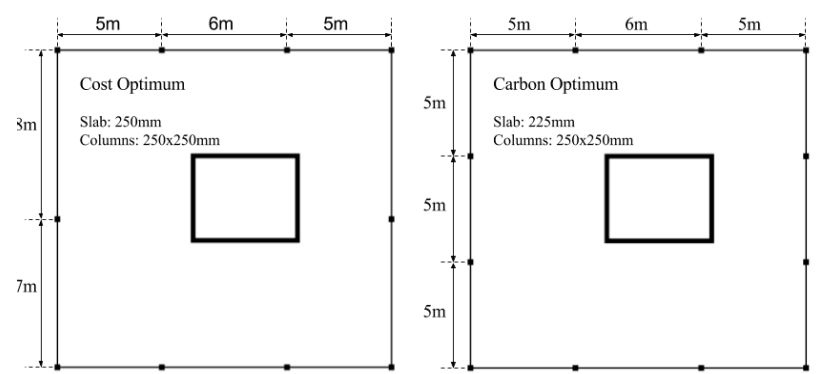

Figure 9. Design configurations that minimise carbon and cost objective functions

In comparison to the carbon optimum design, the columns in the cost optimum solution appear to have a larger impact on the results as the design consists of 10 columns instead of 12 . In addition, the reinforcement rate in the slab is 3\% less than the one in the carbon optimum design. This is due to the thicker slab option $(250 \mathrm{~mm})$.

\subsection{Discussion}

The detailed breakdown of the components in the carbon optimum solution shows the following:

- $66 \%$ in the slab concrete

- $\quad 25 \%$ in the slab reinforcement (basic mesh and additional reinforcement)

- $6 \%$ in the columns concrete

- $3 \%$ in the columns reinforcement

On the othe hand, the detailed breakdown of the components in the cost optimum solution shows the following:

- $25 \%$ in the slab concrete

- $30 \%$ in the slab formwork

- $22 \%$ in the slab reinforcement (basic mesh and additional reinforcement)

- $12 \%$ in the columns reinforcement

- $2 \%$ in the columns concrete

- $\quad 9 \%$ in the columns formwork

The analysis of the two optimum solutions indicates similarities in the final results. The relationship between the cost and the carbon optimum solutions is shown in Figure 10. The cost optimum solution resulted in 111 $\mathrm{kgCO} 2 \mathrm{e} / \mathrm{m}^{2}$ and costs $122 \mathrm{f} / \mathrm{m}^{2}$. On the other hand, the carbon optimum solution costs $126 £ / \mathrm{m}^{2}$ and it has 103 $\mathrm{kgCO} 2 \mathrm{e} / \mathrm{m}^{2}$. The results show an inverse correlation between cost and carbon optimum solutions. The cost optimum design is $3 \%$ cheaper but $7 \%$ more carbon intensive. The obtained results in the tested buiding scenario follow a similar pattern with results found on other structural systems in the literature [8].

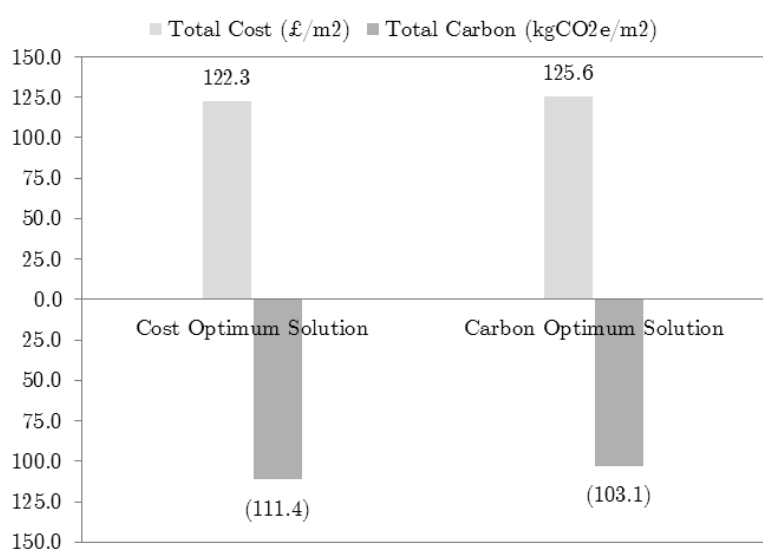

Figure 10. Relationship between cost and carbon optimum solutions

\section{Conclusions}

This study investigates a computational framework for the optimisation of flat slabs in building structures. Three main contributions can be recognised in this research: The first novelty of the study is the use of BIM data combined with a FEM engine that operates within a genetic algorithm solver. The second novelty is the configuration of the optimisation in multiple levels of structural analysis. The final novelty is the development of both cost and carbon functions. A prototypical building scenario is used to validate the methodology. Results show that the effectiveness of the algorithm in finding optimum solutions is not limited by the increased computational complexity of the model. Cost and carbon optimum design configurations were obtained and analysed. For the tested scenario, the cost optimum solution costs only $3 \%$ less than the carbon optimum solution but has $7 \%$ more carbon. The slab accounts for more than $75 \%$ of the total costs in the cost optimum solution when the columns account for approximately $25 \%$. On the other hand, in the carbon optimum design, the slab is responsible for $90 \%$ of the carbon emissions, and only $10 \%$ is related to the columns. Further analysis is required in actual buildings in order to verify the results of the study and evaluate the optimum designs against as built solutions.

\section{Acknowledgements}

This research has been made possible through funding provided by the Engineering and Physical Sciences Research Council (EPSRC) and from Price \& Myers LLP via the UCL EngD Centre (Grant Number: $\mathrm{EP} / \mathrm{G} 037159 / 1)$ in Virtual Environments, Imaging and Visualisation and this is gratefully acknowledged here. 


\section{References}

[1] S. Atabay, "Cost optimization of threedimensional beamless reinforced concrete shear-wall systems via genetic algorithm," Expert Systems with Applications, vol. 36, pp. 3555-3561, 2009.

[2] M. Sahab, A. Ashour and V. Toropov, "Cost optimisation of reinforced concrete flat slab buildings," Engineering Structures, vol. 27, pp. 313-322, 2005.

[3] M. Aldwaik and H. Adeli, "Cost optimization of reinforced concrete flat slabs of arbitrary configuration in irregular highrise building structures," Structural and Multidisciplinary Optimization, vol. 54, pp. 151-164, 2016.

[4] C. Sarma and H. Adeli, "Cost Optimisation of Concrete Structures," Journal of Structural Engineering, vol. 124, pp. 570-578, 1998.

[5] W. Wang, R. Zmeureanu and H. Rivard, "Applying multi-objective genetic algorithms in green building design optimization," Building and Environment, vol. 40, pp. 15121525, 2005.

[6] A. Kosloski, "Cost and Embodied Energy Optimisation of rectangular reinforced concrete beams," Clemson University, Clemson, South Carolina, 2014.

[7] C. Camp and F. Huq, "CO2 and cost optimization of reinforced concrete frames using a big bang-big crunch algorithm," Engineering Structures, vol. 48, pp. 363-372, 2013.

[8] I. Paya-Zaforteza, V. Yepes, A. Hospitaler and F. Gonzalez-Vidosa, "CO2-optimization of reinforced concrete frames by simulated annealing," Engineering Structures, vol. 31, pp. 1501-1508, 2009.

[9] F. Medeiros and M. Kripka, "Optimization of reinforced concrete columns according to different environmental impact assessment parameters," Engineering Structures, vol. 59, pp. 185-194, 2014.

[10] H. Park, B. Kwon, Y. Shin, Y. Kim, T. Hong and S. Choi, "Cost and $\mathrm{CO} 2$ Emission Optimization of Steel Reinforced Concrete Columns in High-Rise Buildings," Energies, vol. 6, no. 11, pp. 5609-5624, 2014.

[11] T. Garcia-Segura, V. Yepes, J. Marti and J. Alcala, "Optimization of concrete I-beams using a new hybrid glowworm swarm algorithm," Latin American Journal of Solids and Structures, vol. 11, no. 7, pp. 1190-1205, 2014.

[12] J. Holland, "Computer programs that "evolve" in ways that resemble natural selection can solve complex problems even their creators do not fully understand," Scientific American, pp. 66-72, 1992.

[13] D. Goldberg, Genetic Algorithms in Search, Optimization and Machine Learning, Boston, MA, USA: Addison-Wesley Longman Publishing Co., Inc., 1989.

[14] Russell-Smith, S., \& Lepech, M. (2011). Dynamic Life Cycle Assessment of Building Design and Retrofit Processes. Computing in Civil Engineering, 760-767.

[15] Eleftheriadis, S., Mumovic, D., \& Greening, P. (2017). Life cycle energy efficiency in building structures: A review of current developments and future outlooks based on BIM capabilities. Renewable and Sustainable Energy Reviews, 811-825 . 